\title{
Evaluation of the effect of static magnetic fields combined with human hepatocyte growth factor on human satellite cell cultures
}

\author{
RICHARD BIRK, ULRICH SOMMER, ANNE FABER, CHRISTOPH ADERHOLD, JOHANNES D. SCHULZ, \\ KARL HÖRMANN, ULRICH REINHART GOESSLER and JENS STERN-STRAETER
}

\author{
Department of Otorhinolaryngology, Head and Neck Surgery, Medical Faculty \\ Mannheim, University of Heidelberg, Mannheim 68167, Germany
}

Received August 7, 2013; Accepted February 18, 2014

DOI: $10.3892 / \mathrm{mmr} .2014 .2083$

\begin{abstract}
Tissue engineering is a promising research field, which aims to create new functional muscle tissue in vitro, by utilizing the myogenic differentiation potential of human stem cells. The objective of the present study was to determine the effect of static magnetic fields (SMF), combined with the use of the myogenic differentiation enhancing hepatocyte growth factor (HGF), on human satellite cell cultures, which are one of the preferred stem cell sources in skeletal muscle tissue engineering. We performed almarBlue ${ }^{\circledR}$ proliferation assays and semi-quantitative reverse transcription polymerase chain reaction (RT-PCR) for the following myogenic markers: desmin (DES), myogenic factor 5 (MYF5), myogenic differentiation antigen 1 (MYOD1), myogenin (MYOG), myosin heavy chain (MYH) and $\alpha 1$ actin (ACTA1) to detect the effects on myogenic maturation. Additionally, immunohistochemical staining (ICC) and fusion index (FI) determination as independent markers of differentiation were performed on satellite cell cultures stimulated with $\mathrm{HGF}$ and $\mathrm{HGF}+\mathrm{SMF}$ with an intensity of $80 \mathrm{mT}$. ICC verified the muscle phenotype at all time points. SMF enhanced the proliferation of satellite cell cultures treated with HGF. RT-PCR analysis, ICC and FI calculation revealed the effects of $\mathrm{HGF} / \mathrm{SMF}$ on the investigated differentiation markers and stimulation with HGF and SMF verified the continuing maturation, however no significant increase in analysed markers could be detected when compared with control cultures treated with serum cessation. In conclusion, $\mathrm{HGF}$ or $\mathrm{HGF}+\mathrm{SMF}$ stimulation of human satellite cell cultures did not lead to the desired enhancement of myogenic maturation of human satellite cell
\end{abstract}

Correspondence to: Dr Jens Stern-Straeter, Department of Otorhinolaryngology, Head and Neck Surgery, Medical Faculty Mannheim, University of Heidelberg, Theodor-Kutzer-Ufer 1-3, 68167 Mannheim, Germany

E-mail: jens.stern-straeter@umm.de

Key words: static magnetic fields, hepatocyte growth factor, skeletal muscle, satellite cells, differentiation cultures compared with cell cultures stimulated with growth factor reduction.

\section{Introduction}

Tissue engineering is a promising field of study in regenerative medicine, which aims to create new functional muscle tissue in vitro using the myogenic differentiation potential of stem cells. Traumatic injuries, tumour excisions or congenital defects often lead to skeletal muscle loss, for which an optimal clinical therapy is lacking. Surgical treatment of these defects with muscle flaps remains a demanding practice that is accompanied by donor-site morbidity. Skeletal muscle tissue engineering aims to overcome this problem with autologous grafting, however it remains an unperfected process due to the difficulty of obtaining sufficient amounts of substitute tissue for functional restoration. Satellite cells, also called myoblasts, are the preferred stem cell for skeletal muscle tissue engineering applications since they can easily be harvested by muscle biopsies, can accurately be characterized and demonstrate a stable differentiation potential into multinucleated myofibers (1). To develop feasible skeletal muscle myotubes in vitro, they have to possess numerous functions and characteristics of skeletal muscle in vivo. Induction of a permanent maturation process in satellite cell cultures remains a challenging procedure that is not completely methodologically sound, however, it is a requirement for sufficient 'neo-tissue' production following autologous transplantation. To cope with this obstacle it is necessary to set up a potent differentiation method, which should not pose any long-term or short-term risks for patients and should be free of possible mutagenic substances (e.g. Matrigel ${ }^{\circledR}$ ). Previously, our group demonstrated that static magnetic fields (SMF) can enhance myogenic differentiation in human satellite cell cultures (1). The effect of SMF on skeletal muscle maturation depends on the concentration of growth factors in the cell culture medium. SMF enhances the maturation of human satellite cells concurrently treated with growth medium (high amounts of growth factors), however, not in cells simultaneously stimulated with serum cessation (differentiation medium, DM; low amount of growth factors) (1). In addition, it has been demonstrated that SMF with an intensity of $80 \mathrm{mT}$ promote myogenic cell differentiation in the immortal rat cell line L6 by increased accumulation of $\alpha$-actin, myosin and formation of large multinucleated myotubes (2). 
The objective of the present study was to determine the effect of SMF combined with the myogenic differentiation enhancing hepatocyte growth factor (HGF) on human satellite cell cultures, one of the preferred stem cell sources in skeletal muscle tissue engineering. HGF is an autocrine growth factor for skeletal muscle satellite cells in vitro (3). It is produced by stromal cells and stimulates epithelial cell proliferation, morphogenesis, motility and angiogenesis in various cell types via tyrosine phosphorylation of its receptor, c-Met (3). Endogenous HGF and the receptor cascade that follows are required for the self repairing of muscle tissue by stimulating the proliferation and activation of satellite cells (3). In human satellite cells, HGF is released following muscle lengthening contraction exercises and regulates satellite cell activation, proliferation and differentiation (4). Therefore, HGF combined with SMF may enhance myogenic differentiation in human satellite cell cultures.

We analysed the maturation process of satellite cells using HGF and SMF as possible promyogenic stimuli by investigating the expression of well-known marker genes that are important during the key steps of myogenesis. Development of multinucleated muscle tissue is accompanied by an upregulation of multiple muscle specific genes and proteins, which can act as specific markers of the different stages of myogenesis (5). As early markers of differentiation we used the transcription factors myogenic factor 5 (MYF5), myogenic differentiation antigen 1 (MYOD1) and myogenin (MYOG), members of the myogenic regulator family (MRF), which serve as important promotors for multiple muscle specific genes, which control the fusion from mononucleated satellite cells into multinucleated myofibers (5). The genes of the contractile apparatus were used as late markers of differentiation. The myosin heavy chain (MYH) is part of the hexameric myosin complex, which consists of four light chains and two heavy chains and constitutes up to $50 \%$ of the total protein content of adult skeletal muscle tissue (6,7). The MYH participates in contraction and can therefore serve as a late marker of differentiation $(7,8)$. As a marker of the terminal stages of myogenesis, $\alpha 1$ actin (ACTA1), an important protein of the contractile apparatus in skeletal muscle tissue, was analysed.

To evaluate the effects of HGF and SMF on human satellite cell cultures, we performed almarBlue ${ }^{\circledR}$ proliferation assays, semi-quantitative reverse transcription polymerase chain reaction (RT-PCR) measurements and immunohistochemical staining (ICC) of the following myogenic markers: MYF5, MYOD1, MYOG, MYH and ACTA1. Additionally, the fusion index (FI) was calculated as an independent marker of myogenic differentiation.

The present study presents data regarding the impact of SMF on satellite cells treated with HGF, considering proliferation and gene and protein expression, which may be attractive to tissue engineering approaches.

\section{Materials and methods}

Cell culture. The Ethics Committee of the Medical Faculty Mannheim, University of Heidelberg (Mannheim, Germany), approved the study protocol and all patients confirmed informed written consent. Satellite cells were isolated from hacked muscle tissue (collected from 15 patients during head and neck surgery) by digestion with collagenase B (Roche Diagnostics, Mannheim, Germany) for $60 \mathrm{~min}$ and $0.05 \%$ trypsin-0.02\% EDTA (PromoCell, Heidelberg, Germany) for $45 \mathrm{~min}$ at $37.5^{\circ} \mathrm{C}$. Next, the cells were filtered through a sterile $70 \mu \mathrm{m}$ cell strainer (Becton-Dickinson, Franklin Lakes, NJ, USA) and purified using the pre-plating technique (9). We pooled the primary myoblasts from the 15 patients and expanded them to passage three. Culture chastity $(>80 \%)$ was established by anti-desmin immunostaining. Cells were grown in $0.2 \%$ gelatine-coated culture flasks (Sigma, Deisenhofen, Germany) in differentiation medium (DM) consisting of minimal essential medium (PromoCell), supplemented with $2 \%$ horse serum (PAA Laboratories, Cölbe, Germany), 2 mM of L-glutamine and penicillin/streptomycin/fungizone (PSF; PromoCell) once they reached $\sim 60 \%$ confluence without addendum, and in addition with $2.5 \mathrm{ng} / \mathrm{ml}$ (stimulation peak) (10) of recombinant human HGF (PeproTech, Rocky Hill, NJ, USA), changed every 72 h. Cells were cultured at $37^{\circ} \mathrm{C}$ in a humidified atmosphere of $5 \% \mathrm{CO}_{2}$ and $95 \%$ air for 1 , $4,8,12,16$ or 21 days.

SMF exposure. SMF were compounded by $4 \times 4 \mathrm{~cm}$ neodymium magnetic plaques with a magnetic field of $80+-5 \mathrm{mT}$, controlled with a gaussmeter. As Coletti et al described, we placed the magnetic plaque $1 \mathrm{~mm}$ beneath the cell monolayer culture (2). The magnetic fields were axial with the magnetic north vector. All cultures were bred concurrently and the experimental conditions were equal with and without SMF.

AlamarBlue ${ }^{\circledR}$ proliferation assay. We plated 5,000 satellite cells per well in $0.2 \%$ gelatine coated 96 -well culture plates. DM (negative control) without addendum and DM + HGF with or without SMF application were analysed. The proliferation was measured at days 1, 4, 8, 16 and 21. We used measurements of existing fluorescence at a wavelength of $540 \mathrm{~nm}$ and absorbance was monitored at $590 \mathrm{~nm}$.

Immunocytochemistry. We performed immunocytochemical characterization on cells grown on chamber culture slides (BD Falcon, Franklin Lakes, NJ, USA). To verify the myogenic differentiation of the cells, we added antibodies directed against desmin (DES; Dako, Hamburg, Germany), MYF5, MYOD1 (Santa Cruz Biotechnology, Inc., Heidelberg, Germany), MYH, (Abcam, Cambridge, UK) and ACTA1 (Zymed Laboratories, Invitrogen, Karlsruhe, Germany). Particular antibody dilutions were DES, 1:100 and MYF5, MYOD1, MYH and ACTA1, 1:50. The special antibodies were followed by a corresponding biotinylated secondary antibody. Chamber culture slides without the first antibody served as the control. We used aminoethylcarbazole (Dako) as a chromogen, to perform the peroxidase reaction. Endogenous peroxidase with $0.3 \%$ hydrogen peroxide for 30 min was used as an inhibitor. In order to inhibit nonspecific antibody reactions, the sections were washed with PBS and incubated with normal sheep serum in PBS for $30 \mathrm{~min}$ at room temperature. Nuclei staining was conducted with Harris haematoxylin. A Zeiss Axiophot microscope (Carl Zeiss, Jena, Germany) served for light microscopic investigations. MYF5 was examined on day 4. DES, MYOD1 and MYH were examined on day 8. 
RNA isolation. For RNA isolation we used the RNA Mini kit (Qiagen, Hilden, Germany), following the manufacturer's instructions. Total RNA concentration was determined by A260 and A280 (A260/A280=1.7-2.0) measurements using an Ultrospec 1000 UV/Visible Spectrophotometer (Amersham Pharmacia Biotech, Buckinghamshire, UK).

cDNA synthesis and PCR. Aliquots of $5 \mu \mathrm{g}$ of total RNA were harvested. We performed reverse transcription using an oligo(dT)-primed first-strand cDNA synthesis kit (Roche Diagnostics), according to the manufacturer's instructions. Using Taq DNA polymerase (Amersham Pharmacia Biotech) and 2-5 $\mu \mathrm{l}$ of reverse transcription products as templates, all cDNA probes were explored for DES, MYF5, MYOD1, MYOG, ACTA1, MYH and GAPDH in a Primus 96 Plus Thermal Cycler (MWG Biotech, Freiburg, Germany) with 30 cycles of PCR. The same primer sequences were used as in our previous study (11). Subsequent electrophoresis was run in $2 \%$ agarose gels containing ethidium bromide. We displayed images of the PCR products under UV light. Relative gene expression was calculated with the densitometry scanning software ImageJ (National Institutes of Health, Bethesda, MD, USA) using GAPDH as the internal standard.

FI determination. To analyse differentiation, the number of nuclei in myotubes was counted and expressed as a percentage of the total number of nuclei analysed. Two co-workers performed the count independently. The FI was determined on day 4 in MYF5-positive myotubes and on day 8 of culture in DES, MYOD and MYH-positive myotubes by dividing the number of nuclei within the myotubes (with two or more nuclei) by the total number of nuclei $x 100$. The nuclei were counterstained with haematoxylin.

\section{Results}

alamarBlue ${ }^{\circledR}$ proliferation assay of human satellite cells treated with $H G F$ and with/without (+/-)SMF.

$H G F$ versus $H G F+S M F$. The fluorescence units (FU) from cultures treated with HGF and SMF were higher at all time points than those cultures treated only with HGF, with the exception of day 21. The values measured for cell cultures stimulated with HGF and SMF were: day 4, 44.15 \pm 1.98 ; day $8,39.33 \pm 1.79$; day $12,43.96 \pm 1.26$; day $16,37.96 \pm 1.92$ and day $21,27.3 \pm 0.8$. The values measured for cultures only treated with HGF following FU were: day 4, 33.84 \pm 2.49 ; day $8,36.97 \pm 1.9$; day $12,42.21 \pm 2.28$; day $16,36.98 \pm 1.13$ and day 21, 29.94 \pm 1.05 (Fig. 1).

Control versus $H G F$. The FU from the cell cultures with the addition of HGF were higher than cell cultures without HGF stimulation on days 4, 12 and 21. The FU detected in cell cultures with HGF treatment were as follows: day 4, $33.84 \pm 2.49$; day $8,36.97 \pm 1.9$; day $12,42.21 \pm 2.28$; day 16 , $36.98 \pm 1.13$ and day $21,29.94 \pm 1.05$. In control cell cultures the results were: day $4,32.53 \pm 0.51$; day $8,37.8 \pm 1.72$; day 12 , $41.36 \pm 1.64$; day $16,36.7 \pm 1.79$ and day $21,27.14 \pm 1.16$ (Fig. 1).

\section{RT-PCR analysis and gene expression}

$D E S$. DES expression was detected in all analysed samples and verified the myogenic phenotype in all cell cultures. DES expression was enhanced by SMF until day 12 in samples

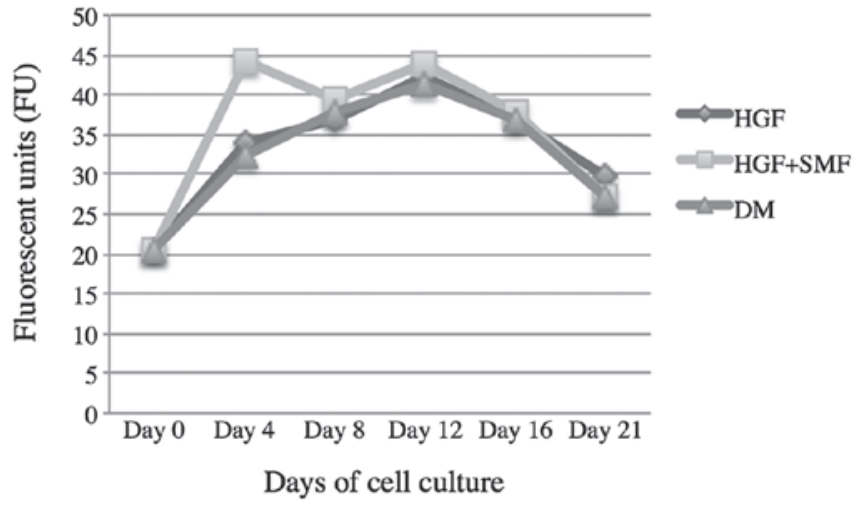

Figure 1. alamarBlue ${ }^{\circledR}$ proliferation assay of human satellite cell cultures stimulated with HGF, HGF + SMF and DM (control cell cultures). HGF, hepatocyte growth factor; SMF, static magnetic fields; DM, differentiation medium.

treated with HGF, as opposed to the cell cultures that were stimulated only with HGF. Following day 12 the effect reversed. HGF and HGF + SMF stimulated human satellite cell cultures demonstrated at all investigated time points, with the exception of day 8 , higher DES expression compared with cultures without stimulation with HGF/SMF (Fig. 2 and 3).

MYF5. MYF5 expression was enhanced until day 8 by SMF application and reduced from day 12-21 compared with cultures stimulated solely by the HGF addendum. The effect of HGF/SMF stimulation on MYF5 expression in human satellite cell cultures was inhibitory taking into account that the highest expression of MYF5 was measured in control cell cultures at day 8, 12 and 21 (Fig. 3).

MYOD1. MYOD1 expression in HGF treated human satellite cells was enhanced in the first days of myogenesis (day 4 and 8) by stimulation with SMF and reduced from day 12-21. The highest expression of MYOD1 in satellite cell cultures without additional stimulation were measured on day 8,16 and 21 (Fig. 3).

MYOG. MRNA levels of MYOG were detected in all groups. SMF treatment decreased MYOG expression in human satellite cell cultures starting from day 4 until day 16 . HGF/SMF stimulation enhanced MYOG expression as of day 12 (Fig. 3).

$M Y H$. SMF application led to a decreased MYH expression in all HGF-treated cell cultures from day 4 until day 16. Stimulation with SMF and/or HGF led to higher mRNA concentrations of MYH from day 12 until day 21 (Fig. 3).

ACTA1. Transcripts of $\alpha$-actin were identified in all analysed cell cultures. The relative expression was reduced by the effect of SMF in HGF-treated human satellite cells at all investigated time points. Treatment with SMF and/or HGF decreased the expression of ACTA1 in human satellite cell cultures compared with cell cultures without stimulation (day 4, 8, 12 and 21; Fig. 3).

Immunohistochemistry and FI. We investigated cell cultures with immunohistochemistry to verify that the primary cells utilised were of muscle origin and did not change phenotype during cultivation by using specific monoclonal antibodies against myogenic markers. All investigated markers were detected in the immunohistochemistry. DES and MYF5 verified the myogenic phenotype. MYF5 was detected on day 4 in 


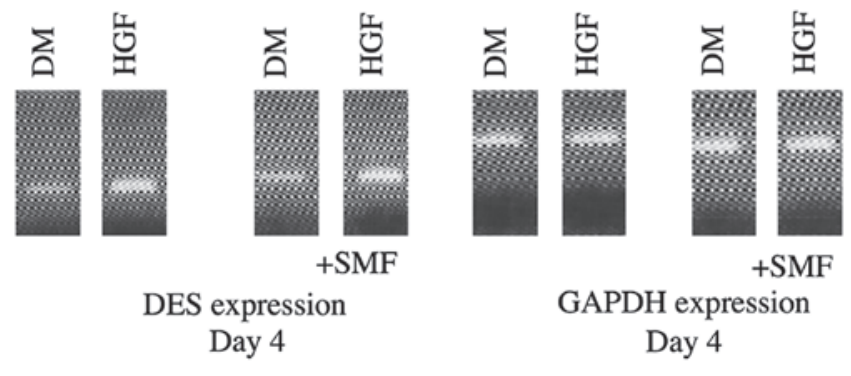

Figure 2. Densitometric quantification of the DES and GAPDH gene expression. GAPDH was used as internal standard. HGF and HGF + SMF led to an increased DES expression in human satellite cell cultures on day 4. DES, desmin; GAPDH, glyceraldehyde 3-phosphate dehydrogenase; HGF, hepatocyte growth factor; SMF, static magnetic fields.

human satellite cell cultures with SMF and/or HGF treatment and without stimulation. No differences between the analysed groups regarding the amount of positively stained cells were detected. ICC of DES demonstrated more positively stained cells in SMF-treated cell cultures on day 8, verifying the gene expression results (Fig. 4). FI in HGF-treated DES-positive myofibers $(0.5653)$ was lower on day 8 than in those treated with SMF (0.6294), supporting the gene expression results. The myogenic differentiation of human satellite cells was confirmed by detection of the myogenic markers MYOD1 and MYH. No significant differences between tested groups were observed regarding the amounts of positively stained cells. On day 8 MYOD1-positive cells demonstrated higher FI in the negative control cultures (0.7380) than in myotubes treated with HGF (0.55). At no time could we detect any contracting myofibrils.

\section{Discussion}

Skeletal muscle tissue engineering aims to generate new functional muscle tissue in vitro by utilising the myogenic differentiation potential of stem cells. A prerequisite for clinical application are strong and harmless differentiation stimuli that can induce permanent maturation in expanded human stem cells. Two promising stimuli which have been demonstrated to enhance skeletal muscle differentiation under certain culture conditions are HGF and SMF $(1,4)$. However, the impact of SMF on myogenic progenitor cells is debated and highly depends on cell origin and the strength of the magnetic field. It has been demonstrated that SMF with an intensity of $80 \mathrm{mT}$ promote myogenic cell differentiation in the immortal rat cell line L6 by increased accumulation of actin, myosin and the formation of large multinucleated myotubes (2). Conversely, in vitro the reduction of the earth magnetic field to $0.3 \mathrm{mT}$ leads to the inhibition of proliferation and differentiation of skeletal muscle in newborn rat satellite cell cultures, while low-intensity magnetic fields (60-160 mT) display a stimulatory effect that leads to an increased formation rate of myotubes (12). Sakurai et al demonstrated that the exposure to strong SMF (10 T) led to significant numbers of orientated myotubes in cultures from the mouse-derived myoblast cell line (C2C12). No effect on differentiation was observed when $3 \mathrm{~T}$ was applied (13). Furthermore, Kim et al demonstrated that SMF (2 T) in C2C12 myoblast cells inhibits proliferation and may delay cell growth by altering the subcellular localization of gamma complex protein 3 (14). Our group demonstrated that the effect of SMF with $80 \mathrm{mT}$ on human satellite cells depends on the concentration of growth factors in the cell culture medium (1). Therefore, we postulated that SMF combined with HGF could enhance myogenic differentiation in human satellite cell cultures. HGF is an autocrine secreted growth factor that stimulates satellite cell differentiation via tyrosine phosphorylation of its receptor c-Met (4). The present study demonstrates the first results, to the best of our knowledge, regarding the effect of HGF on human satellite cell cultures with and without the combination of SMF exposure.

Proliferation analysis using the alamarBlue ${ }^{\circledR}$ assay revealed that $\mathrm{HGF}$, with a cell culture media concentration of $2.5 \mathrm{ng} / \mathrm{ml}$, does not lead to a significant increase of cell proliferation in human satellite cell cultures. The measurements of the FU demonstrated slightly higher proliferation rates only on days 4, 12 and 21 compared with non-stimulated cell cultures. This finding stands in contrast to the results of Allen et al who demonstrated that, in rat skeletal muscle satellite cells, proliferation is stimulated by HGF with a peak concentration of $2.5 \mathrm{ng} / \mathrm{ml}(3,15)$. This finding supports the importance of utilising primary human stem cells in tissue engineering research and indicates that findings from other cell origins cannot necessarily be transferred for human tissue engineering applications. Higher concentrations of HGF (e.g. $50 \mathrm{ng} / \mathrm{ml}$ ) inhibit proliferation and drive rat satellite cells back to quiescence by the induction of myostatin expression (10). Therefore, we did not enhance the HGF concentration in the culture media. High rates of proliferation antagonize the differentiation of myoblasts into myotubes as withdrawal from the cell cycle is essential to initiate the differentiation cascade. Additional SMF stimulation combined with HGF treatment led to a slight increase in the proliferation of human satellite cell cultures, with the exception of day 21. This is an interesting finding, which provides evidence that SMF may enhance the activity of HGF in human satellite cell cultures. Scientific understanding of the regulatory effects of SMF on cell biology are not fully understood and further investigations are required to decipher them. Stimulation of SMF alone has been demonstrated by us and other research groups to have no stimulatory effect on cell proliferation in human satellite cell cultures (1).

DES is one of the earliest muscle-specific proteins to be expressed during myogenesis. It is an intermediate filament, which is part of the cytoskeleton (16). It can therefore act as an early marker of myogenic differentiation. When compared with non-stimulated cell cultures, gene expression measurements of DES revealed that HGF and HGF + SMF stimulation lead to an augmentation of DES expression in human satellite cell cultures, indicating that more cells are driven into the myogenic differentiation lineage by this treatment. During the early course of myogenesis, SMF + HGF enhanced DES expression in human satellite cell cultures, compared with cultures solely treated with HGF, indicating that SMF may enhance the pro-myogenic effect of HGF at the beginning of the maturation process. This finding is supported by the calculations of the FI, which were higher in cell cultures treated with SMF + HGF compared with HGF-treated cell cultures on day 8 in DES positively stained myofibers. 
A

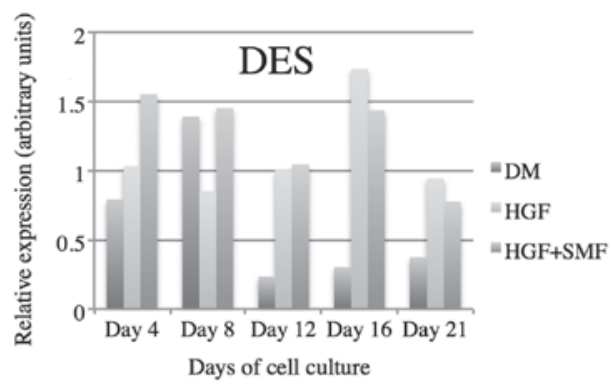

C

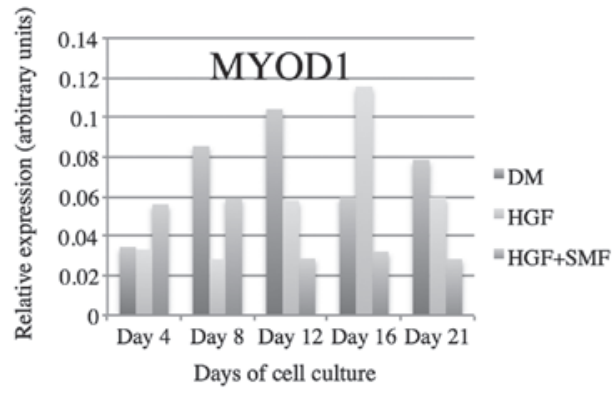

$\mathbf{E}$

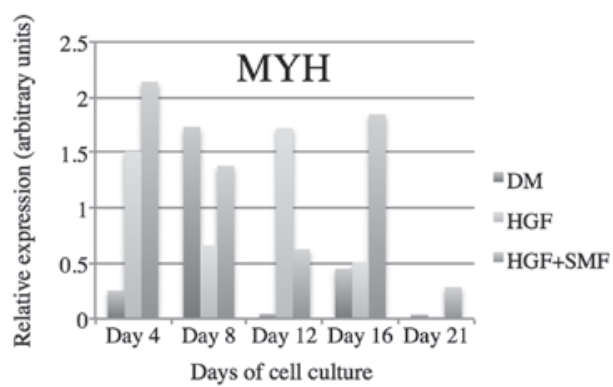

B

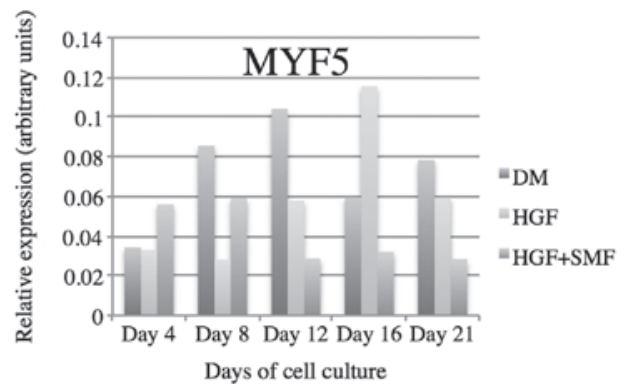

D

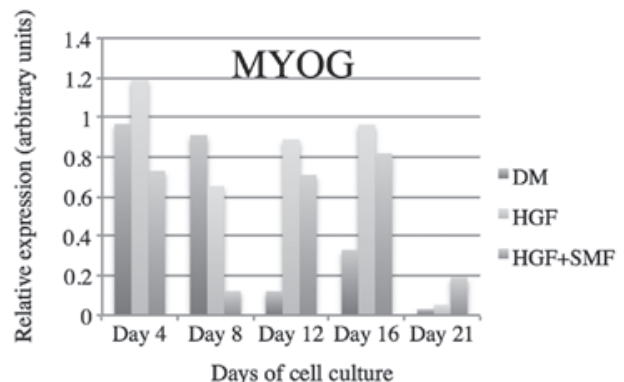

$\mathbf{F}$

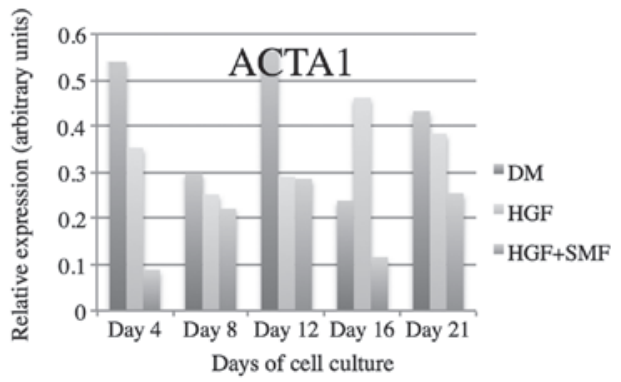

Figure 3. Gene expression analyses of (A) DES, (B) MYF5, (C) MYOD1, (D) MYOG, (E) MYH and (F) ACTA1 in human satellite cell cultures stimulated with HGF, HGF + SMF and without additional stimulation (DM). GAPDH served as a reference gene. DES, desmin; GAPDH, glyceraldehyde 3-phosphate dehydrogenase; HGF, hepatocyte growth factor; SMF, static magnetic fields; MYF5, myogenic factor 5; MYOD1, myogenic differentiation antigen 1; MYOG, myogenin; MYH, myosin heavy chain; ACTA1, $\alpha 1$ actin; DM, differentiation medium.

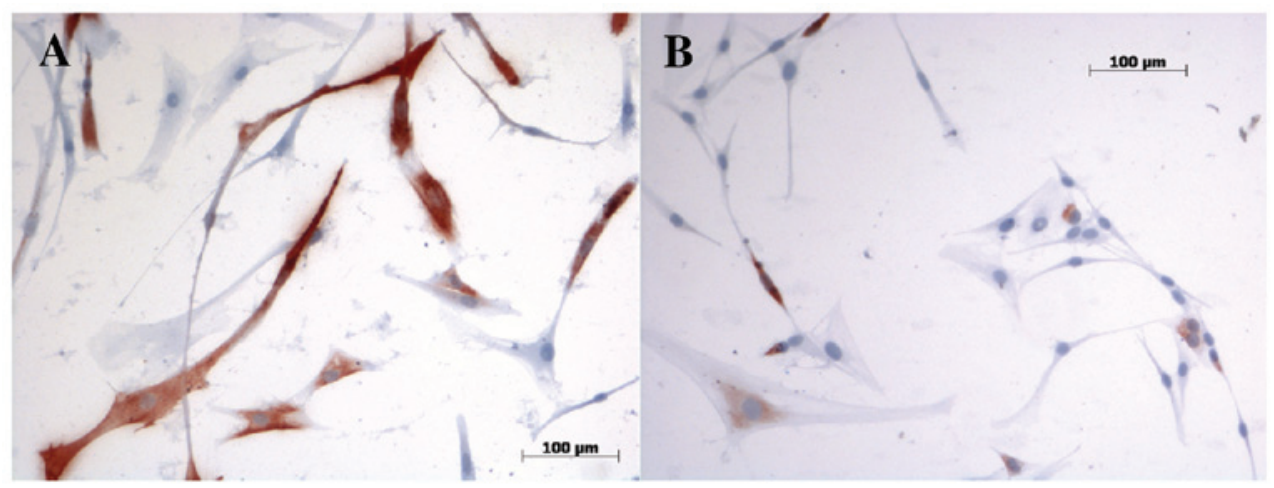

Figure 4. Example of the immunohistochemical staining of DES in HGF stimulated cell cultures on day 8 (A) with and (B) without SMF treatment. DES desmin; HGF, hepatocyte growth factor; SMF, static magnetic fields.

Another early marker of satellite cell maturation is the myogenic regulatory factor MYF5, which is necessary for myogenic cell specialization and is upregulated during the early course of differentiation $(1,17)$. Gene expression analysis of MYF5 demonstrated that only the combination of SMF and
HGF lead to an augmentation of gene expression during the very early events of myogenesis (day 4) supporting the theory that SMF may intensify the effects of HGF. As time went on this effect could no longer be identified. Control cell cultures demonstrated higher MYF5 expression as of day 8. MYF5 was 
also detected by ICC on day 4 , however no significant differences between the investigated groups could be detected.

The myogenic determination factor MYOD1 is a transcription factor which induces differentiation by promoting multiple muscle specific genes by heterodimerization with the E-proteins. It crosstalks with the cell cycle regulators, which leads to the withdrawal of the cell cycle, a prerequisite for myogenic differentiation $(18,19)$. The upregulation of MYOD1 represents the start of myogenic differentiation (1). Gene expression analysis of MYOD1 revealed an inhibitory effect of HGF and HGF + SMF starting on day 8 in human satellite cell cultures. This finding is supported by the calculations of the FI, which demonstrated a higher FI in non-stimulated human satellite cell cultures compared with HGF stimulated cultures. At the beginning of the differentiation process (day 4), SMF + HGF stimulation led to an increased MYOD1 expression, emphasising the possibility that the combination of SMF and HGF may enhance myogenic differentiation at the beginning of myogenesis.

Compared with non-stimulated cell cultures, gene expression measurements of MYOG, a transcription factor of the MRF family that operates during the development of myotubes $(1,20)$, demonstrated that the stimulation of human satellite cell cultures with HGF and SMF + HGF led to a higher MYOG gene expression starting from day 12 . The stimulating effect of HGF was suppressed by additional treatment with SMF. This finding is in accordance with our previously obtained results, in which we demonstrated that SMF stimulation leads to lower expression levels of MYOG gene expression (1).

The MYH is part of the contractile apparatus and exists in multiple isoforms that can be used to characterise the fibre type and developmental status of human satellite cell cultures $(1,21)$. It serves as a late marker of myogenic differentiation. Gene expression analysis demonstrated that stimulation with HGF and $\mathrm{SMF}+\mathrm{HGF}$ led to an increase in MYH gene expression starting on day 12, indicating a higher degree of maturation in the late phase of myogenesis. Additional stimulation with SMF did not enhance the gene expression of MYH in HGF-treated cell cultures. ICC experiments could detect the $\mathrm{MYH}$, however no differences between the groups were observed.

ACTA1 is an important structural protein of the contractile apparatus in skeletal muscle tissue. Along with myosin, it is responsible for muscle contraction. It can also act as a marker of differentiation for the final stages of muscle differentiation $(22,23)$. Gene expression experiments revealed that $\mathrm{HGF}$ and $\mathrm{HGF}+\mathrm{SMF}$ stimulation lead to a downregulation of the ACTA1 gene at all investigated time points. The relative expression is additionally reduced by the effect of SMF. This finding is in accordance with our previously obtained results, which demonstrated that ACTA1 expression in human satellite cell cultures decreased following SMF stimulation, indicating a lower degree of maturation following SMF stimulation (1). This finding stands in opposition with the results of Coletti et al, who described increased accommodation of $\alpha$-actin in rat satellite cell cultures following SMF stimulation (2). This discrepancy can be explained by the different origins of utilised cells (rat vs human) and emphasises the importance of employing primary human cells in tissue engineering research.
In summary, we conclude that the stimulation of human satellite cell cultures with $\mathrm{HGF}$ or $\mathrm{HGF}+\mathrm{SMF}$ does not lead to the desired enhancement of myogenic differentiation in terms of increased myotube formation and generation of contractile muscle tissue. Marker gene expression analysis revealed heterogeneous results for the different myogenic markers of differentiation, thus no categorical statements regarding the effects of HGF and HGF + SMF on the maturation of human satellite cell cultures can be made. Further investigations are required to explore the additional effects of SMF on human satellite cells used for tissue engineering.

\section{Acknowledgements}

The authors would like to thank Michael Collins for his help with the study and Petra Prohaska for her excellent technical assistance.

\section{References}

1. Stern-Straeter J, Bonaterra GA, Kassner SS, et al: Impact of static magnetic fields on human myoblast cell cultures. Int J Mol Med 28: 907-917, 2011.

2. Coletti D, Teodori L, Albertini MC, et al: Static magnetic fields enhance skeletal muscle differentiation in vitro by improving myoblast alignment. Cytometry A 71: 846-856, 2007.

3. Sheehan SM, Tatsumi R, Temm-Grove CJ and Allen RE: HGF is an autocrine growth factor for skeletal muscle satellite cells in vitro. Muscle Nerve 23: 239-245, 2000.

4. O'Reilly C, McKay B, Phillips S, Tarnopolsky M and Parise G: Hepatocyte growth factor (HGF) and the satellite cell response following muscle lengthening contractions in humans. Muscle Nerve 38: 1434-1442, 2008.

5. Tapscott SJ and Weintraub H: MyoD and the regulation of myogenesis by helix-loop-helix proteins. J Clin Invest 87: 1133-1138, 1991.

6. Wright C, Haddad F, Qin AX and Baldwin KM: Analysis of myosin heavy chain mRNA expression by RT-PCR. J Appl Physiol (1985) 83: 1389-1396, 1997.

7. Stern-Straeter J, Bonaterra GA, Kassner SS, et al: Characterization of human myoblast differentiation for tissue-engineering purposes by quantitative gene expression analysis. J Tissue Eng Regen Med 5: e197-e206, 2011.

8. Wehrle U, Düsterhöft S and Pette D: Effects of chronic electrical stimulation on myosin heavy chain expression in satellite cell cultures derived from rat muscles of different fiber-type composition. Differentiation 58: 37-46, 1994.

9. Machida S, Spangenburg EE and Booth FW: Primary rat muscle progenitor cells have decreased proliferation and myotube formation during passages. Cell Prolif 37: 267-277, 2004.

10. Yamada M, Tatsumi R, Yamanouchi K, et al: High concentrations of HGF inhibit skeletal muscle satellite cell proliferation in vitro by inducing expression of myostatin: a possible mechanism for reestablishing satellite cell quiescence in vivo. Am J Physiol Cell Physiol 298: C465-C476, 2010.

11. Stern-Straeter J, Bran G, Riedel F, Sauter A, Hörmann K and Goessler UR: Characterization of human myoblast cultures for tissue engineering. Int J Mol Med 21: 49-56, 2008.

12. Eldashev IS, Shchegolev BF, Surma SV and Belostotskaia GB: Effect of low-intensity magnetic fields on the development of satellite muscle cells of a newborn rat in the primary culture. Biofizika 55: 868-874, 2010 (In Russian).

13. Sakurai T, Hashimoto A, Kiyokawa T, Kikuchi K and Miyakoshi J: Myotube orientation using strong static magnetic fields. Bioelectromagnetics 33: 421-427, 2012.

14. Kim S and Im W: Static magnetic fields inhibit proliferation and disperse subcellular localization of gamma complex protein 3 in cultured C2C12 myoblast cells. Cell Biochem Biophys 57: 1-8, 2010.

15. Allen RE, Sheehan SM, Taylor RG, Kendall TL and Rice GM: Hepatocyte growth factor activates quiescent skeletal muscle satellite cells in vitro. J Cell Physiol 165: 307-312, 1995. 
16. Lazarides E: Intermediate filaments: a chemically heterogeneous, developmentally regulated class of proteins. Annu Rev Biochem 51: 219-250, 1982.

17. Cosgrove BD, Sacco A, Gilbert PM and Blau HM: A home away from home: challenges and opportunities in engineering in vitro muscle satellite cell niches. Differentiation 78: 185-194, 2009.

18. Kitzmann M and Fernandez A: Crosstalk between cell cycle regulators and the myogenic factor MyoD in skeletal myoblasts. Cell Mol Life Sci 58: 571-579, 2001

19. Kataoka Y, Matsumura I, Ezoe S, et al: Reciprocal inhibition between MyoD and STAT3 in the regulation of growth and differentiation of myoblasts. J Biol Chem 278: 44178-44187, 2003.
20. Sassoon DA: Myogenic regulatory factors: dissecting their role and regulation during vertebrate embryogenesis. Dev Biol 156: $11-23,1993$

21. Pette D and Staron RS: Myosin isoforms, muscle fiber types, and transitions. Microsc Res Tech 50: 500-509, 2000.

22. Laing NG, Dye DE, Wallgren-Pettersson C, et al:: Mutations and polymorphisms of the skeletal muscle alpha-actin gene (ACTA1). Hum Mutat 30: 1267-1277, 2009.

23. Smith CK 2nd, Janney MJ and Allen RE: Temporal expression of myogenic regulatory genes during activation, proliferation, and differentiation of rat skeletal muscle satellite cells. J Cell Physiol 159: 379-385, 1994. 